\title{
Gravitational Waves from Neutron Stars: A Review
}

\author{
Paul D. Lasky \\ Monash Centre for Astrophysics, School of Physics and Astronomy, Monash University, VIC 3800, Australia \\ Email: paul.lasky@monash.edu
}

(Received August 20, 2015; ACCEPTED August 26, 2015)

\begin{abstract}
Neutron stars are excellent emitters of gravitational waves. Squeezing matter beyond nuclear densities invites exotic physical processes, many of which violently transfer large amounts of mass at relativistic velocities, disrupting spacetime and generating copious quantities of gravitational radiation. I review mechanisms for generating gravitational waves with neutron stars. This includes gravitational waves from radio and millisecond pulsars, magnetars, accreting systems, and newly born neutron stars, with mechanisms including magnetic and thermoelastic deformations, various stellar oscillation modes, and core superfluid turbulence. I also focus on what physics can be learnt from a gravitational wave detection, and where additional research is required to fully understand the dominant physical processes at play.
\end{abstract}

Keywords: gravitational waves - stars: neutron

\section{INTRODUCTION}

The dawn of gravitational wave astronomy is one of the most anticipated scientific advances of the coming decade. The second generation, ground-based gravitational wave interferometers, Advanced Laser Interferometer Gravitational-wave Observatory (aLIGO Aasi et al. 2015b) and Virgo (Acernese et al. 2015), are due to start observing later in 2015 and in 2016 , respectively. The first aLIGO observing run is expected to be a few times more sensitive than initial LIGO, with a full order of magnitude increase in strain sensitivity by $\sim 2019$ (for a review, see Aasi et al. 2013).

The inspiral and merger of compact binary systems (neutron stars and/or black holes) are commonly expected to be the first detections with aLIGO. The most robust predictions for event rates come from observations of the binary neutron star population within our galaxy, with an expected binary neutron-star detection rate for the aLIGO/Virgo network at full sensitivity between 0.4 and 400 per yr (Abadie et al. 2010b). But there are many other exciting astrophysical and cosmological sources of gravitational waves (for a brief review, see Riles 2013). Loosely, these can be divided into four categories: compact binary coalescences, bursts, stochastic backgrounds, and continuous waves. Burst sources include gravitational waves generated in nearby supernova explosions, magnetar flares, and cosmic string cusps. Stochastic backgrounds arise from the incoherent sum of sources throughout the Universe, including from compact binary systems, rotating neutron stars, and primordial perturbations during inflation. Continuous gravitational waves are almost monochromatic signals generated typically by rotating, nonaxisymmetric neutron stars.

The above laundry list of gravitational wave sources prominently features neutron stars in their many guises. While supranuclear densities, relativistic velocities, and enormous magnetic fields are exactly what makes neutron stars amenable to emitting gravitational waves of sufficient amplitude to be detectable on Earth, it is also these qualities that makes it difficult to provide accurate predictions of the gravitational wave amplitudes, and hence detectability, of their signals. A positive gravitational wave detection from a neutron star would engender great excitement, but it is the potential to understand the interior structure of neutron stars that will make this field truly revolutionary.

In this review, I provide a detailed overview of many proposed gravitational wave generation mechanisms in neutron stars, including state-of-the-art estimates of the gravitational wave detectability. These include gravitational waves generated from magnetic deformations in newly-born and older isolated radio pulsars (Section 2), accreting systems (Section 3 ), impulsive and continuous-wave emission from pulsar glitches (Section 4), magnetar flares (Section 5), and from superfluid turbulence in the stellar cores (Section 6). As well as discussing gravitational wave detectability, I also concentrate on what physics can be learnt from a future, positive gravitational wave detection.

It is worth stressing that this is not a review of all gravitational wave sources in the audio band, but is instead designed 

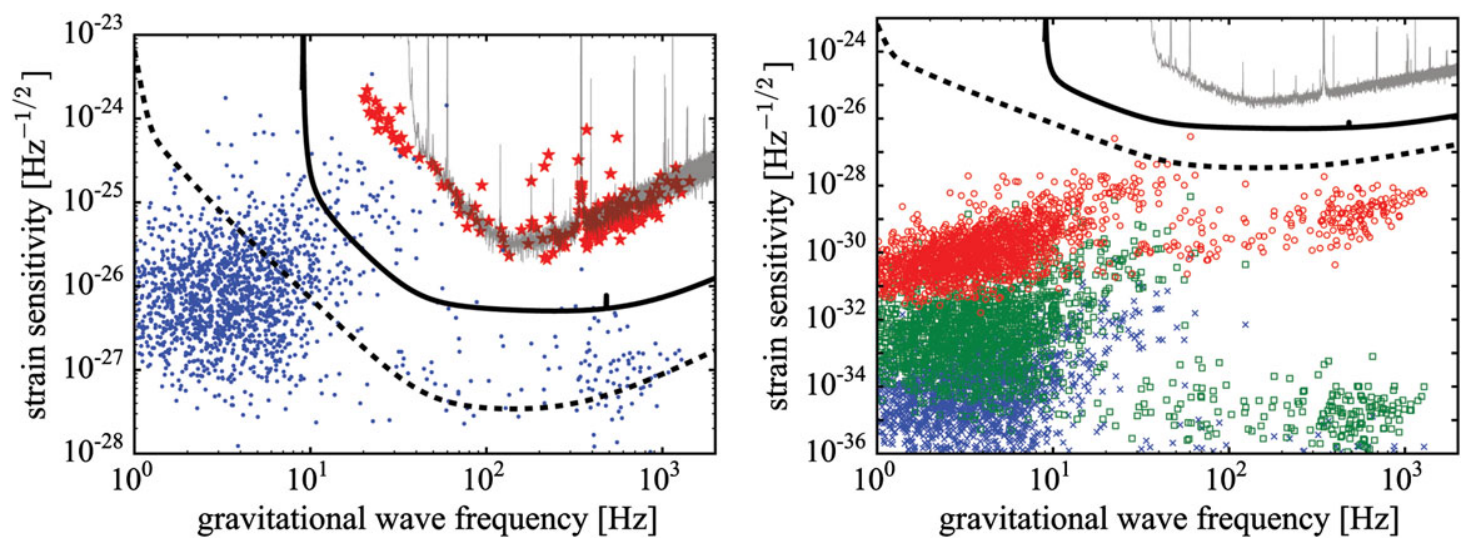

Figure 1. Left panel: Current upper limits on the gravitational wave strain from known pulsars (red stars; data from Aasi et al. 2014a) and the spin down limits for known pulsars in the Australia Telescope National Facility (ATNF) catalogue (blue dots). Right panel: Gravitational wave strain predictions for known pulsars. The blue crosses and green squares are for normal neutron star matter with purely poloidal magnetic fields and $\Lambda=0.01$, respectively [see Equation (2) and surrounding text]. The red circles assume the neutron stars are colour-flavour-locked phase [CFL; Equation (3)] with $\langle B\rangle=10 B_{\mathrm{p}}$. In both figures, the solid and dashed black curves show the projected strain sensitivity for aLIGO and ET respectively, and the grey curve is the strain sensitivity for the initial S5 run assuming a two-year coherent integration (e.g., see Dupuis \& Woan 2005).

to review the theory of gravitational wave sources from neutron stars. The paper is but one in a series highlighting Australia's contribution to gravitational wave research (Howell et al., in preparation; Kerr, in preparation; Slagmolen, in preparation).

\section{MAGNETIC DEFORMATIONS}

Spinning neutron stars possessing asymmetric deformations emit gravitational waves. Such deformations can be generated through elastic strains in the crust (Bildsten 1998; Ushomirsky, Cutler, \& Bildsten 2000), or strong magnetic fields in the core (Bonazzola \& Gourgoulhon 1996). A triaxial body rotating about one of its principal moments of inertia will emit radiation at frequency $f_{\mathrm{gw}}=2 v$, where $v$ is the star's spin frequency. More precisely, a freely precessing, axisymmetric body, with principal moment of inertia, $I_{\mathrm{zz}}$, and equatorial ellipticity, $\epsilon$, emits a characteristic gravitational wave strain (Zimmerman \& Szedenits 1979)

$$
\begin{aligned}
h_{0} & =\frac{4 \pi^{2} G}{c^{4}} \frac{I_{z z} f_{\mathrm{gw}}^{2} \epsilon}{d} \\
& =4.2 \times 10^{-26}\left(\frac{\epsilon}{10^{-6}}\right)\left(\frac{P}{10 \mathrm{~ms}}\right)^{-2}\left(\frac{d}{1 \mathrm{kpc}}\right)^{-1},
\end{aligned}
$$

where $d$ is the distance to the source. For comparison, the smallest, upper limit on stellar ellipticity for young neutron stars in supernova remnants comes from LIGO observations of Vela Jr. (G266.2-1.2), with $\epsilon \leq 2.3 \times 10^{-7}$ (Aasi et al. 2014b). The overall ellipticity record-holder is for the millisecond pulsars J2124-3358 and J2129-5721 with $\epsilon \leq 6.7 \times 10^{-8}$ and $\epsilon \leq 6.8 \times 10^{-8}$, respectively (Aasi et al. 2014a). Typical ellipticity constraints for isolated radio pulsars are $\epsilon \lesssim 10^{-4}-10^{-6}$ (Aasi et al. 2014a).

\subsection{Spin-down limit}

An absolute upper limit on the gravitational wave strain from individual pulsars, known as the spin-down limit, can be calculated assuming the observed loss of rotational energy is all going into gravitational radiation. The left-hand panel of Figure 1 shows the current upper limits on the gravitational wave strain from a search for known pulsars (red stars; Aasi et al. 2014a), as well as the spin down limits for the known pulsars in the ATNF catalogue (Manchester et al. 2005) ${ }^{1}$. The observed gravitational wave upper limits beat the spin-down limit for both the Crab and Vela pulsars, while a further five pulsars are within a factor of five. Also plotted in Figure 1 are the projected strain sensitivities for aLIGO and ET (solid- and dashed-black curves respectively), and the strain sensitivity for the S5 run of initial LIGO (for details, see Aasi et al. 2014a). Age-based upper limits on the gravitational wave strain can also be calculated for neutron stars with unknown spin frequencies (Wette et al. 2008).

\subsection{Magnetic field-induced ellipticities}

As a general rule of thumb, a neutron star's ellipticity scales with the square of the volume-averaged magnetic field inside the star, $\langle B\rangle$ (e.g., Haskell et al. 2008), implying the characteristic gravitational wave strain also scales as $h_{0} \sim\langle B\rangle^{2}$. The dipole, poloidal component of the magnetic field at the surface of the star is inferred from the star's spin period and its derivative, but very little is known about the interior field strength and/or configuration.

A considerable body of work has been devoted to understanding possible magnetic field configurations ${ }^{2}$, in part to

\footnotetext{
${ }^{1}$ http://www.atnf.csiro.au/people/pulsar/psrcat/

${ }^{2}$ In Australia, work on possible magnetic field configurations in the cores of stars dates back to the 1960 's, where Monaghan $(1965,1966)$ calculated
} 
answer the question of gravitational wave detectability, but also to understand the inverse problem regarding what can be learnt from future gravitational wave observations. Purely poloidal and purely toroidal fields are known to be unstable on dynamical timescales (e.g., Wright 1973; Braithwaite \& Spruit 2006; Braithwaite 2007). Mixed fields, where a toroidal component threads the closed-field-line region of the poloidal field, commonly termed 'twisted-torus' fields, are generally believed to be dynamically stable (e.g., Braithwaite \& Nordlund 2006; Ciolfi et al. 2009; Akgün et al. 2013), although it has been suggested this is dependent on the equation of state (Lander \& Jones 2012; Mitchell et al. 2015). Moreover, a recent study of the effect twisted-torus fields have on crust-core rotational coupling during neutron star spin down suggest these fields may be unstable on a spin down timescale (Glampedakis \& Lasky 2015).

While generating solutions to the equations of Newtonian and general relativistic magnetohydrostatics is a noble task, it is no substitute for understanding the initial-value problem that brings one towards realistic magnetic field configurations. In Newtonian simulations with soft equations of state (typically more applicable to main-sequence stars than to neutron stars), Braithwaite and co. showed magnetic fields evolve either to axisymmetric, twisted-tori (Braithwaite \& Nordlund 2006; Braithwaite 2009), or nonaxisymmetric configurations (Braithwaite 2008), depending on the initial conditions. General relativistic magnetohydrodynamic simulations ubiquitously show the development of non-axisymmetries (Kiuchi, Yoshida, \& Shibata 2011; Lasky et al. 2011; Lasky, Zink, \& Kokkotas 2012; Ciolfi et al. 2011; Ciolfi \& Rezzolla 2012), however these simulations begin with a restricted set of initial conditions. The long-term evolution to stable equilibria in these systems is still an open question.

The aforementioned uncertainty in possible magnetic field configurations translates to uncertainty in gravitational wave predictions. The relative strengths of poloidal and toroidal components strongly affects the stellar ellipticity, and hence gravitational wave detectability (Haskell et al. 2008; Colaiuda et al. 2008; Ciolfi et al. 2009; Mastrano et al. 2011). Relatively standard models suggest (e.g., Mastrano et al. 2011)

$$
\epsilon \approx 4.5 \times 10^{-7}\left(\frac{B_{\mathrm{p}}}{10^{14} \mathrm{G}}\right)^{2}\left(1-\frac{0.389}{\Lambda}\right)
$$

where $B_{\mathrm{p}}$ is the poloidal component of the surface magnetic field, $\Lambda$ is the ratio of poloidal-to-total magnetic field energy (i.e., $0 \leq \Lambda \leq 1$, with $\Lambda=0,1$ corresponding to purely toroidal and purely poloidal fields repsectively), and I have normalised to fiducial values of stellar mass and radius. Unfortunately, Equation (2) is pessimistic for gravitational wave detection with aLIGO as the only neutron stars with $B_{\mathrm{p}} \sim 10^{14} \mathrm{G}$ are the magnetars, but with spin periods of

magnetic field equilibria in polytropes, which even included calculations of magnetic-field induced stellar ellipticities.
$P \sim 1-10$ s they emit gravitational waves at frequencies too low. The right- hand panel of Figure 1 shows predictions for the gravitational wave strain for known pulsars from the ATNF pulsar catalogue (Manchester et al. 2005). The blue crosses assume a purely poloidal field i.e., Equation (2) with $\Lambda=1$, while the green squares assume a strong internal toroidal component given by $\Lambda=0.01$. The red circles are described below, but with normal neutron star matter the prospects for gravitational wave detection from magnetic field-induced non-axisymmetries are grim. One therefore has to hope that Nature has been kind, and has provided neutron stars with strong internal, toroidal components of the magnetic field.

\subsection{Generating large ellipticities}

Certainly, one expects newly born neutron stars to have large internal toroidal fields and correspondingly large ellipticities; strong differential rotation combined with turbulent convection in the nascent neutron star drives an $\alpha-\Omega$ dynamo, which winds up a toroidal field as strong as $\sim 10^{16} \mathrm{G}$ (e.g., Duncan \& Thompson 1992). Although the symmetry axis of the wound-up field is aligned to the spin axis of the star, the ellipsoidal star will evolve on a viscous dissipation timescale to become an orthogonal rotator through free-body precession, and hence optimal emitter of gravitational waves (Cutler 2002; Stella et al. 2005; Dall'Osso, Shore, \& Stella 2009). How such a strong field evolves over secular timescales is an open question.

A recent spate of papers has shown that strong toroidal fields can also be achieved in systems in dynamical equilibrium by prescribing different forms for the azimuthal currents in the star (Ciolfi \& Rezzolla 2013), and also by invoking stratified, two fluid stellar models (i.e., where the neutrons form a superfluid condensate and the protons are either a normal fluid or a superconductor; Glampedakis, Andersson, \& Lander 2012a; Lander 2013). Interestingly, exotic states of matter in the core such as crystalline coloursuperconductors allow for significantly higher ellipticities (Owen 2005; Haskell et al. 2007). Recently, Glampedakis, Jones, \& Samuelsson (2012b) showed that, if the ground state of neutron star matter is a colour-superconductor, then the colour-magnetic vortex tension force leads to significantly larger mountains than for normal proton superconductors. They derived fiducial ellipticities for purely poloidal fields of $^{3}$

$$
\begin{gathered}
\epsilon^{2 \mathrm{SC}} \approx 4.0 \times 10^{-6} \frac{\langle B\rangle}{10^{14} \mathrm{G}}, \\
\epsilon^{\mathrm{CFL}} \approx 1.2 \times 10^{-5} \frac{\langle B\rangle}{10^{14} \mathrm{G}},
\end{gathered}
$$

\footnotetext{
${ }^{3}$ It is worth noting that, in any kind of superconductor, the ellipticity scales linearly with the volume-averaged magnetic field (Cutler 2002), cf. $\langle B\rangle^{2}$ for normal matter.
} 
where $\epsilon^{2 \mathrm{SC}}$ and $\epsilon^{\mathrm{CFL}}$ respectively denote the ellipticities if matter is in a two-flavour phase (i.e., only the $u$ and $d$ quarks form superconducting pairs) and a colour-flavourlocked phase (where all three quark species are paired), and $\langle B\rangle$ is the volume averaged magnetic field in the core of the star. The red circles in Figure 1 show gravitational wave predictions for a colour-flavour locked superconductor, Equation (3), with the average internal field ten times the observationally inferred surface field, i.e., $\langle B\rangle=10 B_{\mathrm{p}}$. These results are fascinating; a positive detection of gravitational waves from magnetic deformations in neutron stars is a fundamental probe of the fundamental state of nuclear matter.

It is worth mentioning that the discussion in this section pertains to the optimal case of a rigidly rotating, axisymmetric body rotating about one of its principal moments of inertia. Such a body emits monochromatic gravitational waves at a frequency of $2 v$. If the neutron star core contains a pinned superfluid, it will emit also at the spin frequency, $v$ (Jones 2010). In general, a non-aligned rotator will emit at $v, 2 v$, and a number of frequencies straddling these values (Zimmerman 1980; Jones \& Andersson 2002; van den Broeck 2005; Lasky $\&$ Melatos 2013). For radio pulsars, such modulations would also be present in other observables such as pulse time-ofarrivals and radio polarisation (Jones \& Andersson 2001; Jones \& Andersson 2002).

\subsection{Oscillations in young neutron stars}

The strongest emitters of gravitational waves from magnetic deformations are likely young, rapidly rotating neutron stars. Such stars may also emit through other channels, the most likely being the unstable $r$-mode, whose restoring force is the Coriolis force; see Section 3.2 for a detailed description of the physics of $r$-modes. Targeted gravitational wave searches of young neutron stars in supernova remnants can be adapted to set limits on the amplitude of such oscillations (Owen 2010). This was first done with a 12-day coherent search of S5 LIGO data targeting the neutron star in the supernova remnant Cassiopeia A (Wette et al. 2008; Abadie et al. 2010a). The search has since been extended to nine young supernova remnants, with the most sensitive $r$-mode fractional amplitude being less than $4 \times 10^{-5}$ for Vela Jr. (Aasi et al. 2014b). Such a limit is encroaching 'interesting values' of the amplitude when compared to simulations that calculate the non-linear saturation amplitude of various $r$-modes in young neutron stars (Bondarescu, Teukolsky, \& Wasserman 2009; Aasi et al. 2014b).

\section{ACCRETING SYSTEMS}

\subsection{Torque balance}

An observational conundrum drives research into gravitational wave emission from accreting systems; namely, the absence of accreting pulsars with spin frequencies $v \gtrsim 700 \mathrm{~Hz}$
(Chakrabarty et al. 2003; Patruno 2010). The argument is simple: measured accretion rates allow one to calculate the angular momentum being transferred to the neutron star, which should be spun up to frequencies at, or near, the breakup frequency ( $v \gtrsim 1 \mathrm{kHz}$ for most equations of state Cook, Shapiro, \& Teukolsky 1994). The common explanation is that the neutron stars are losing angular momentum through the emission of gravitational radiation (Papaloizou \& Pringle 1978; Wagoner 1984; Bildsten 1998), although alternatives exist, most notably invoking interactions between the accretion disk and the companion star's magnetic field (e.g., White \& Zhang 1997; Patruno, Haskell, \& D'Angelo 2012).

Balancing the accretion torques with gravitational wave emission allows one to estimate an upper limit on the gravitational wave strain independent of the emission mechanism (Wagoner 1984):

$$
h_{0}^{\mathrm{EQ}}=5.5 \times 10^{-27} \frac{R_{10}^{3 / 4}}{M_{1.4}^{1 / 4}}\left(\frac{F_{X}}{F_{\star}}\right)^{1 / 2}\left(\frac{300 \mathrm{~Hz}}{v}\right)^{1 / 2},
$$

where $R_{10}=R / 10 \mathrm{~km}, M_{1.4}=M / 1.4 M_{\odot}, F_{X}$ is the X-ray flux, and $F_{\star}=10^{-8} \mathrm{erg} \mathrm{cm}^{-2} \mathrm{~s}^{-1}$. The $v^{-1 / 2}$ scaling arises only because of the spin-equilibrium assumption; slower rotators need larger ellipticities for torque balance. If one builds a mountain without being in spin equilibrium, then the gravitational wave strain is simply given by Equation (1).

Equation (5) implies the loudest gravitational wave emitters are the brightest $\mathrm{X}$-ray sources such as the low-mass $\mathrm{X}$-ray binaries, the brightest of which being Scorpius X-1 (Sco X-1). Watts et al. (2008) utilised observations of Sco $\mathrm{X}-1$ and other known accreting systems to determine their gravitational wave detectability with current and future interferometers showing that, even at torque balance, most systems will be very difficult to detect with aLIGO.

The LIGO Scientific Collaboration has given periodic gravitational waves from Sco X-1 high priority, with multiple data analysis pipelines already fully developed (see Messenger et al. 2015, and references therein). The unknown spin period of the neutron star in Sco X-1 and the variable accretion rate that drives spin-wandering of the neutron star complicate these searches, but the best gravitational wave upper limit utilises the Sideband search (Sammut et al. 2014), which gives a $95 \%$ upper limit of $h_{0} \lesssim 8 \times 10^{-25} \mathrm{~Hz}$ at 150 $\mathrm{Hz}$ (Aasi et al. 2015a). This is still above the torque balance limit for Sco X-1, $h_{0}^{\mathrm{EQ}} \approx 3.5 \times 10^{-26}(300 \mathrm{~Hz} / v)^{1 / 2}$, but this is expected to be beaten with aLIGO observations (Whelan et al. 2015).

Torque balance is an empirically derived limit that is independent of the mechanism generating the gravitational radiation. From a theoretical perspective, there are a number of ways in which sufficient energy can be lost to gravitational waves, including unstable oscillation modes (Andersson 1998), and non-axisymmetric deformations supported by the magnetic field (Cutler 2002; Melatos \& Payne 2005) or elastic crust (Bildsten 1998; Ushomirsky et al. 2000). 

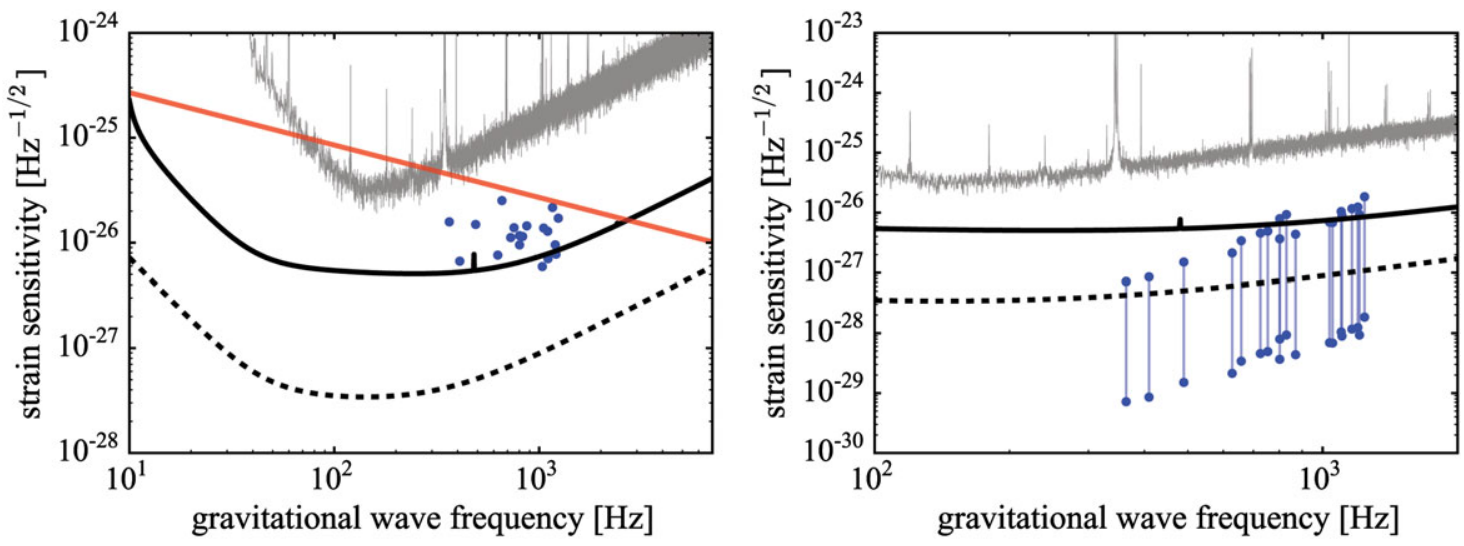

Figure 2. Left panel: Gravitational wave torque balance limit for known accreting millisecond pulsars and systems with burst oscillations assuming gravitational wave emission at twice the neutron star spin period (blue dots). Also shown is the torque balance limit for Sco X-1 (red curve) which has unknown spin period. Data is collated from Watts et al. (2008) and Haskell et al. (2015). Right panel: Gravitational wave predictions for magnetic mountains on known accreting X-ray pulsars, where the range is for magnetic field strengths at the onset of accretion of between $B_{\star}=10^{10}$ and $10^{12} \mathrm{G}$ (for details of the calculation, see Haskell et al. 2015). In both panes, the solid- and dashed-black curves show the projected strain sensitivity for aLIGO and ET respectively, and the grey curve is the strain sensitivity for the initial S5 run, assuming two years of coherent integration time. For comparison, current observational upper limits on Sco X1 from LIGO are $\lesssim 8 \times 10^{-25}$ at $150 \mathrm{~Hz}$, which utilise a 10-day, semi-coherent analysis (Aasi et al. 2015a).

The torque balance limit for accreting systems with known spin periods is shown in the left-hand panel of Figure 2 (blue dots), and for Sco X-1 (red curve) which has an unknown spin period. The LIGO, aLIGO, and ET sensitivity curves in this figure assume two years integration; see Watts et al. (2008) for more detailed, realistic estimates of signal-tonoise ratios of these systems. Note that current upper limits of gravitational wave emission from Sco X-1 utilise a semicoherent, 10-day search, which therefore yields less stringent upper limits than the LIGO curve in Figure 2 (Aasi et al. 2015a).

\subsection{Unstable oscillation modes}

Unstable oscillations modes, in particular $r$-modes, have received significant attention as potential sources of detectable gravitational waves. These inertial modes - toroidal modes of oscillation for which the restoring force is the Coriolis force - are generically unstable to a phenomena known as the Chandrasekhar-Friedman-Schutz (CFS) instability (Chandrasekhar 1970; Friedman \& Schutz 1978). Consider an oscillation mode in a rotating star. The rotation induces both forward and backward propagating modes, but the backward propagating modes are being dragged forward by the star's rotation. Although this retrograde mode continues to move backwards in the star's rotating frame, if the star is rotating sufficiently fast it will be prograde in the inertial frame. The mode loses energy to gravitational waves, but carries with it positive angular momentum from the star. This positive angular momentum is subtracted from the negative angular momentum of the mode, which becomes more negative, growing the amplitude of oscillation. As the mode grows, it emits more positive angular momentum, and continues to grow even faster. Hence, a sufficiently rapidly rotating star is unstable to gravitational wave emission.

At all stellar rotation rates, $r$-modes are retrograde in the co-moving frame, but prograde in the inertial frame, implying they are generically unstable to the CFS instability (Andersson \& Kokkotas 1998; Friedman \& Morsink 1998). A struggle therefore ensues between the gravitational waves which drive up the oscillation amplitude and the viscous damping that acts to suppress the mode amplitude. There exists a narrow range in spin period and temperature for which gravitational wave emission dominates over viscous dissipation: at low temperatures, $T \lesssim$ (few) $\times 10^{9} \mathrm{~K}$, viscous dissipation is dominated by shear viscosity, while at high temperatures, $T \gtrsim$ (few) $\times 10^{9} \mathrm{~K}$, bulk viscosity is the main culprit. Details of the $r$-mode instability window therefore depend sensitively on relatively unknown neutron star physics, including microphysics and complicated crust-core interactions (for a review, see Andersson \& Kokkotas 2001). This somewhat simple picture describing the $r$-mode instability window is inconsistent with observed spins and temperatures of lowmass X-ray binaries, implying the complete physical picture behind this mechanism is ill-understood (Ho, Andersson, \& Haskell 2011; Haskell, Degenaar, \& Ho 2012).

Strohmayer \& Mahmoodifar (2014) recently analysed Rossi X-ray Timing Explorer (RXTF) observations of the accreting millisecond pulsar XTE J1751-305, finding evidence for a coherent oscillation mode during outburst that they attributed to either an $r$ - or $g$-mode (for which buoyancy is the restoring force). The $r$-mode interpretation is the most interesting in the context of gravitational wave emission; had the outburst occurred during the S5 LIGO run it would have been marginally observable (Andersson, Jones, \& Ho 2014). However, doubt has been cast over the $r$-mode interpretation; Andersson et al. (2014) also showed that the mode amplitude 
required to interpret the observations necessarily leads to large spin-down of the neutron star, which is inconsistent with the neutron star's observed spin evolution.

\subsection{Mountains}

On the other hand, permanent non-axisymmetries supported magnetically or elastically may generate a significant gravitational wave signature. Accreted matter accumulates on the neutron star, is buried, compressed, and undergoes a range of nuclear reactions (e.g., Haensel \& Zdunik 1990). In the non-magnetic case, asymmetries in the accretion lead to compositional and heating asymmetries, which induce stellar deformations. Approximate expressions for the quadrupolar deformation can be derived in terms of the quadrupolar component of the temperature variation and reaction threshold energies (Ushomirsky et al. 2000). Such deformations are limited by the maximum stress the crust can sustain before breaking (Ushomirsky et al. 2000; Haskell, Jones, \& Andersson 2006; Johnson-McDaniel \& Owen 2013), which typically gives larger gravitational wave estimates than torque balance (Haskell et al. 2015).

Accretion also affects the structure of the neutron star's magnetic field which, in turn, changes the accretion dynamics. The magnetosphere funnels accreted matter onto the poles, at which point it spreads towards the equator, dragging, compressing, and burying the local stellar magnetic field (Hameury et al. 1983; Melatos \& Phinney 2001). Such fields can lead to stellar deformations considerably larger than those due to the background magnetic field inferred from the external, dipole (Payne \& Melatos 2004; Melatos \& Payne 2005; Vigelius \& Melatos 2009), which may even generate gravitational waves detectable by aLIGO or the Einstein Telescope (Priymak, Melatos, \& Payne 2011), particularly if the buried field is $B \gtrsim 10^{12} \mathrm{G}$ (Haskell et al. 2015). This is shown in the right-hand panel of Figure 2, where the gravitational wave signal from accreting systems with known spin periods is shown assuming initial magnetic fields of $B=10^{10}$ and $10^{12} \mathrm{G}$ (for details of this calculation, see Haskell et al. 2015). Finally, it is worth remarking that, given a positive gravitational wave detection, an in-principle measurement of cyclotron resonant scattering features in the X-ray spectrum may be able to discern magnetic or elastic mountains (Priymak, Melatos, \& Lasky 2014; Haskell et al. 2015).

\section{PULSAR GLITCHES}

Pulsar glitches are sudden jumps in the neutron star spin frequency with wide-ranging fractional amplitudes, $10^{-11} \lesssim$ $\Delta v / v \lesssim 10^{-4}$ (Melatos et al. 2008; Espinoza et al. 2011; Espinoza et al. 2014). The exact mechanism driving a glitch is not fully understood, although it is clear that a sudden transfer of angular momentum occurs between the rapidly rotating superfluid interior and the outer crust (for a recent review, see Haskell \& Melatos 2015). Such large angular momentum transfer lends itself to the generation of gravitational radia- tion through a variety of avenues, as both broadband burst emission from the glitch and as a continuous wave signal during the glitch recovery phase.

\subsection{Gravitational wave bursts}

Most theories of pulsar glitches rely on the general mechanism introduced by Anderson \& Itoh (1975). The quantum mechanical nature of superfluids implies the neutron star's rotation is attributed to an array of $\sim 10^{18}$ quantised superfluid vortices. These thread the entire star, but are pinned to lattice sites and/or crustal defects, and hence are restricted from moving outwards as the crust spins down through the usual electromagnetic torques. The superfluid core therefore retains a higher angular velocity than the crust of the star, and a differential lag builds up between these two components. A glitch occurs when $\sim 10^{7}-10^{15}$ vortices catastrophically unpin and move outwards, thereby rapidly transferring angular momentum to the crust.

That so many vortices are required to unpin simultaneously implies an avalanche trigger process must be at work. Such an avalanche is likely non-axisymmetric, and hence capable of emitting gravitational waves through a variety of channels. Warszawski \& Melatos (2012) simulated the motion of vortices, showing that a burst of gravitational waves emitted through the current quadrupole has a characteristic strain

$$
\begin{aligned}
h_{0} & \approx 10^{-24}\left(\frac{\Delta \Omega / \Omega}{10^{-7}}\right)\left(\frac{\Omega}{10^{2} \mathrm{rad} \mathrm{s}^{-1}}\right)^{3} \\
& \times\left(\frac{\Delta r}{10^{-2} \mathrm{~m}}\right)^{-1}\left(\frac{d}{1 \mathrm{kpc}}\right)^{-1},
\end{aligned}
$$

where $\Delta r$ is the average distance travelled by a vortex during a glitch. The non-detection of gravitational waves from a glitch in the Vela pulsar during the fifth LIGO Science run in 2006 put an upper limit of $h_{0} \lesssim 10^{-20}$ (Abadie et al. 2011b), implying an upper limit of $\Delta r \lesssim 10^{-2} \mathrm{~m}$ and a lower limit on the glitch duration $\gtrsim 10^{-4} \mathrm{~ms}$.

Figure 3 shows a histogram of estimates for the gravitational wave strain using Equation (6), where the glitches are taken from the ATNF glitch catalogue (Manchester et al. $2005)^{4}$ The vertical-dashed line shows the empirical gravitational wave strain upper limit from the August 2006 glitch of the Vela pulsar (Abadie et al. 2011b).

\subsection{Glitch recovery}

The motion of vortices is expected to excite hydrodynamic oscillation modes such as $f$ - and $p$-modes, for which the pressure is the restoring force, and inertial $r$-modes (e.g., Andersson 1998; Andersson \& Kokkotas 1998; Glampedakis \& Andersson 2009). Although $r$-modes are expected to be the dominant emission mechanism, recent estimates of their detectability with ground-based interferometers is pessimistic

\footnotetext{
${ }^{4}$ http://www.atnf.csiro.au/people/pulsar/psrcat/glitchTbl.html
} 


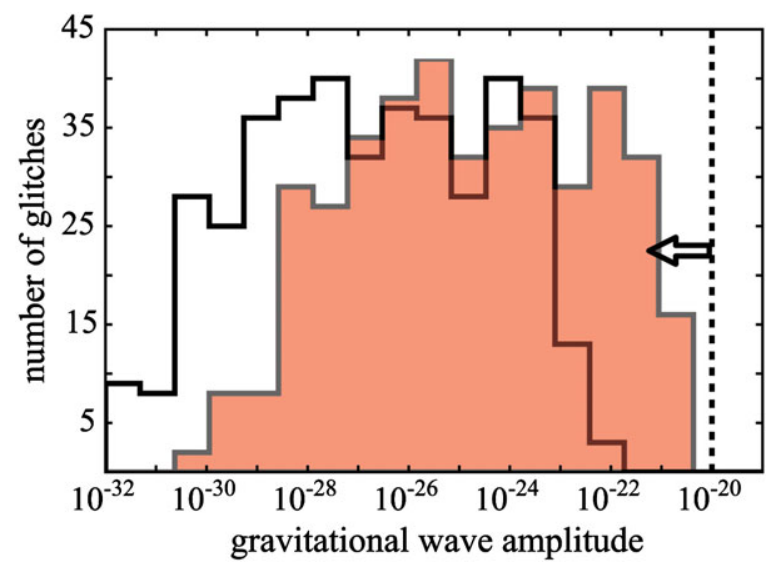

Figure 3. Predicted gravitational wave amplitudes for known glitches using Equation (6) with $\Delta r=10^{-2} m$ (black, unfilled histogram) and $\Delta r=10^{-4}$ (red, filled histogram). The vertical, dashed line is the gravitational wave strain upper limit derived for the August 2006 glitch of the Vela pulsar during the LIGO S5 run (Abadie et al. 2011b)

(Sidery, Passamonti, \& Andersson 2010). More optimistic estimates have been provided for gravitational waves from meridional Ekman flows that are expected to couple the core and crust rotation post-glitch (van Eysden \& Melatos 2008; Bennett, van Eysden, \& Melatos 2010), although it is worth noting the model is sensitive to many unknown, dimensionless parameters such as the Ekman number, normalised compressibility, and Brunt-Väisälä frequency. Moreover, strong stratification may suppress Ekman flows to thin boundary layers in the outer regions of the star (Abney \& Epstein 1996; Melatos 2012), thereby reducing the gravitational wave strain estimates. Finally, it has recently been suggested that vortex avalanches that are believed to trigger glitches leave behind long-lived, large-scale inhomogeneities on the vortex distribution that are potentially observable by aLIGO (Melatos, Douglass, \& Simula 2015).

\section{MAGNETAR FLARES}

Perhaps, the most exotic of all neutron stars are the magnetars; isolated neutron stars with external dipole magnetic fields $B_{p} \gtrsim 10^{14} \mathrm{G}$. The decay of the immense magnetic field powers irregular bursts in hard X-rays and soft $\gamma$-rays, with typical peak luminosities $\sim 10^{38}-10^{43} \mathrm{erg} \mathrm{s}^{-1}$. Three $\mathrm{gi}^{-}$ ant flares have been observed in our Galaxy ${ }^{5}$, the largest of which was the extreme outburst from SGR 1806-20 on 2004 December 27 which emitted a total, isotropic energy of $2 \times 10^{46}$ erg (Palmer et al. 2005).

Giant flares are commonly attributed to catastrophic rearrangements of the magnetic field, either internal or external to the neutron star (e.g., Duncan \& Thompson 1992; Thomp-

\footnotetext{
${ }^{5}$ Interestingly, LIGO non-detections of gravitational waves from nearby short-duration, hard-spectrum gamma-ray bursts GRB 051103 (Abbott et al. 2008a) and GRB 070201 (Abadie et al. 2012) rule out compact binary coalescences as their progenitors, implying these are most likely extragalactic magnetar giant flares.
}

son \& Duncan 1995). Strong coupling between the magnetic field and the solid crust imply the latter will stress, and potentially rupture (although, see Levin \& Lyutikov 2012), exciting oscillation modes in the star's crust and core. Observations of quasi-periodic oscillations (QPOs) in the tails of two giant flares (Israel et al. 2005; Strohmayer \& Watts 2005) has engendered excitement about the potential new field of neutron star asteroseismology. In particular, the interpretation of QPOs as stellar magneto-elastic oscillations provide enticing potential to infer neutron star structural parameters (see Levin 2006; Glampedakis, Samuelsson, \& Andersson 2006; Levin 2007; Sotani, Kokkotas, \& Stergioulas 2007; Sotani, Colaiuda, \& Kokkotas 2008; Colaiuda, Beyer, \& Kokkotas 2009; van Hoven \& Levin 2011; Levin \& van Hoven 2011; Gabler et al. 2013, 2014; Huppenkothen, Watts, \& Levin 2014 c, and references therein).

\subsection{KiloHertz gravitational waves}

First attempts to understand gravitational-wave emission from magnetar flares were optimistic. Ioka (2001) assumed an instantaneous change in the star's moment of inertia from a rearrangement of the global, internal magnetic field can excite the neutron star's fundamental $f$ mode (typically at frequencies, $f \sim 1-2 \mathrm{kHz}$ ), a study that was backed up by Corsi \& Owen (2011). These studies estimated that the energy in gravitational waves could be $E_{\mathrm{gw}} \sim 10^{48}-10^{49} \mathrm{erg}$, comparable to the energy emitted in electromagnetic waves, and certainly detectable in the Advanced Detector Era.

In contrast, Levin \& van Hoven (2011) showed that, while the order-of-magnitude estimates of Ioka (2001) and Corsi \& Owen (2011) are correct, in practice there is poor energy conversion into the $f$-mode, and prospects for gravitational wave detection in the Advanced Era are pessimistic. The analytic calculation of Levin \& van Hoven (2011) looked at the energy conversion from a flare triggered in the star's magnetosphere. Zink, Lasky, \& Kokkotas (2012) and Ciolfi \& Rezzolla (2012) performed complementary, general relativistic magnetohydrodynamic simulations using their respective codes (Lasky et al. 2011; Ciolfi et al. 2011) for catastrophic reconfigurations of the internal magnetic field, also finding that the $f$-mode is not sufficiently excited to generate a detectable gravitational wave signature. Although these works had different predictions for the gravitational wave scaling as a function of magnetic field strength, they both predicted approximately 10 orders of magnitude less energy being emitted in gravitational waves than Corsi \& Owen (2011).

Figure 4 shows a histogram of the possible gravitational wave energies emitted in the $f$-mode from known galactic magnetars ${ }^{6}$ using the calculations of Ciolfi \& Rezzolla (2012) (shaded red histogram) and Zink et al. (2012); Lasky et al. (2012) (empty black histogram). In blue are the gravitational

\footnotetext{
${ }^{6}$ The known magnetars are taken from the McGill Magnetar catalogue (Olausen \& Kaspi 2014).
} 


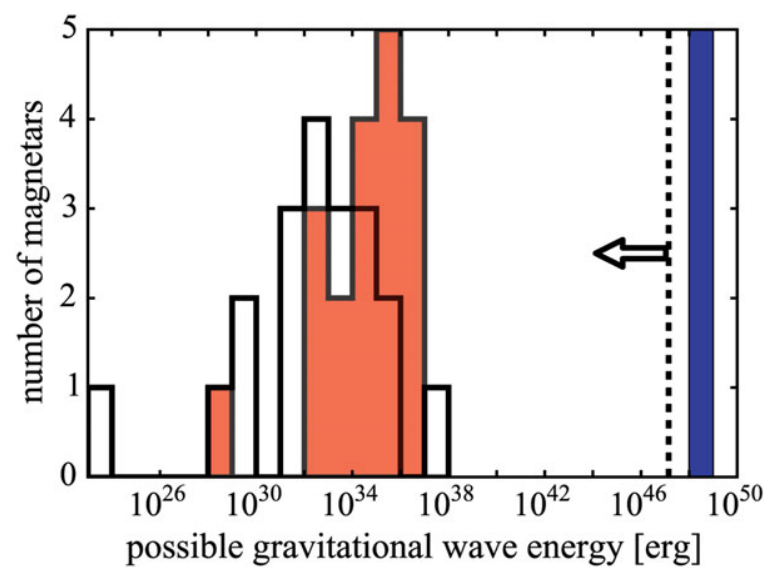

Figure 4. Predictions of the possible gravitational wave energy emitted in $f$-mode oscillations if giant flares were to go off in each observed galactic magnetar given the calculations of (Ciolfi \& Rezzolla 2012, shaded red histogram) and (Zink et al. 2012; Lasky et al. 2012, empty black histogram). Plotted in blue are the optimistic predictions of Ioka (2001) and Corsi \& Owen (2011), and the dashed black line is the upper limit on the $f$-mode gravitational wave energy from the giant flare from SGR 1806-20 (Abadie et al. 2011a).

wave predictions from Ioka (2001) and Corsi \& Owen (2011), while the dashed black line gives the observed upper limit on the $f$-mode gravitational wave energy from the 2004 giant flare in SGR 1806-20 (Abadie et al. 2011a, see below).

\subsection{Low-frequency gravitational waves}

Although the $f$-mode couples well to gravitational radiation, one of the reasons it is not an optimal source for groundbased detectors is that it is damped through the emission of gravitational radiation in $\lesssim 0.1 \mathrm{~s}$ (Detweiler 1975; McDermott, van Horn, \& Hansen 1988). Longer lasting, lower frequency modes excited from magnetar flares have been suggested as potential sources of detectable gravitational waves (Kashiyama \& Ioka 2011; Zink et al. 2012; Lasky et al. 2012). To generate sufficient mass motions, and therefore a detectable gravitational wave signal, these modes need to be global core oscillations, and hence are unlikely the cause of the observed QPOs. A key uncertainty for these low frequency modes is their damping time, which underpins their gravitational wave emission. If these modes form an Alfvén continuum of frequencies, where the QPOs arise as the edges or turning points of the continuum (Levin 2007), then resonant absorption should quickly redistribute energy, resulting in short lifetimes $(\lesssim 1 \mathrm{~s})$ for the QPOs (Levin 2007; Levin \& van Hoven 2011; Gabler et al. 2011; van Hoven \& Levin 2012; Huppenkothen et al. 2014c), and pessimistic estimates for gravitational wave emission. On the other hand, if global Alfvén waves are excited in the core (Zink et al. 2012) then they may live for 100 s of seconds, and be potentially detectable by third-generation interferometers, or even aLIGO in the case of extremely strong $\left(\sim 10^{16} \mathrm{G}\right)$ magnetic fields in the core (Glampedakis \& Jones 2014).

\subsection{Current observational limits}

At the time of the giant flare from SGR 1806-20, the $4 \mathrm{~km}$ Hanford detectors was the only interferometer operating. An upper limit of $E_{\mathrm{gw}}<7.7 \times 10^{46} \mathrm{erg}$ was achieved around the $92.5 \mathrm{~Hz}$ QPO, with a significantly weaker constraint placed in the $\mathrm{kHz}$ range where one expects the $f$-mode. The most sensitive gravitational wave search of the giant flare was from the AURIGA bar detector (Baggio et al. 2005). They searched for exponentially decaying signals (with decay time $0.1 \mathrm{~s}$ ) in a small frequency band around $900 \mathrm{~Hz}$. Subsequent LIGO/Virgo searches of regular flares from magnetars have yielded comparable gravitational wave limits to the original 2004 burst (Abbott et al. 2008b; Abadie et al. 2011a).

While galactic giant flares only occur approximately once per decade, the regularity of magnetar flare storms implies they may provide an attractive alternative. If such flares also excite normal stellar modes, it is easy to imagine that stacking gravitational wave data from multiple flares could allow for sufficient integration time. Indeed, such a gravitational wave search has been designed (Kalmus et al. 2009) and implemented to search for gravitational waves in LIGO/Virgo data (Abbott et al. 2009). These attempts have further been buoyed by the successful detection of QPOs in ordinary magnetar flares (Huppenkothen et al. 2014a, 2014b).

\section{SUPERFLUID TURBULENCE}

Soon after the realisation that neutron star matter should form a superfluid condensate (Baym, Pethick, \& Pines 1969, and references therein), Greenstein (1970) suggested this superfluid should be in a highly turbulent state ${ }^{7}$. Such turbulence can be either hydrodynamic (e.g., Peralta et al. 2005, 2006a, 2006b, 2008) or quantum mechanical (e.g., Tsubota 2009; Andersson, Comer, \& Prix 2003; Mastrano \& Melatos 2005; Andersson, Sidery, \& Comer 2007; Link 2012a, 2012b). Regardless, the turbulence is likely driven by differential rotation between the superfluid core (with Reynolds number $\operatorname{Re} \sim 10^{11}$ ) and the solid crust that is being slowly spun down by electromagnetic torques.

\subsection{Individual sources}

Although the turbulence is axisymmetric when averaged over long times, it is instantaneously non-axisymmetric, and therefore emits stochastic gravitational waves. The signal was first calculated in Peralta et al. (2006a) by numerically solving the spherical Couette-flow problem with $\operatorname{Re} \lesssim 10^{6}$. A subsequent analytic calculation (Melatos \& Peralta 2010) made a more robust prediction of the gravitational wave amplitude and spectrum, showing in particular that the peak of the gravitational wave signal occurs near the inverse of the turbulence decoherence timescale, $\tau_{c}$. For reasonable neutron

\footnotetext{
${ }^{7}$ The beautifully succinct abstract is seldom seen today: 'The neutron superfluid in most neutron stars should be in a highly turbulent state. If so, this turbulence drastically alters its rotational properties' (Greenstein 1970).
} 


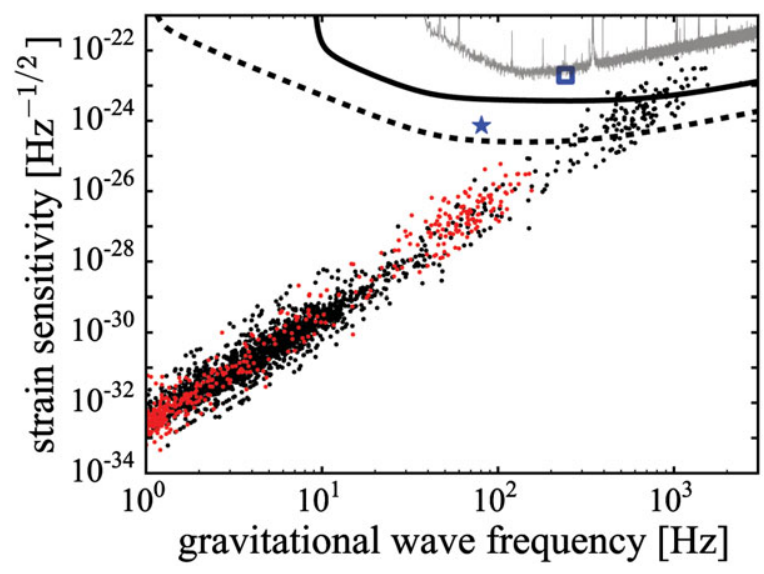

Figure 5. Peak gravitational wave amplitudes from superfluid turbulence from galactic pulsars with Ro $=\Delta \Omega / \Omega=10^{-1}$ (black points) and $10^{-2}$ (red points). The blue star and square are hypothetical, nearby $(d=10 \mathrm{pc})$ rapid rotators with spin periods $p=3 \mathrm{~ms}$ and $10 \mathrm{~ms}$, and $\mathrm{Ro}=10^{-2}$ and $10^{-1}$, respectively.

star parameters, this decoherence timescale is

$$
\tau_{c} \approx 26\left(\frac{\Delta \Omega}{10 \mathrm{rad} \mathrm{s}^{-1}}\right)^{-1} \mathrm{~ms},
$$

where $\Delta \Omega$ is the difference in angular frequency between the crust and the core of the neutron star, which can potentially be related to the star's angular frequency, $\Omega$, through the Rossby number, Ro $=\Delta \Omega / \Omega$. For many pulsars, this places the peak of the gravitational wave signal in LIGO's most sensitive band, although it is worth noting there is considerable uncertainty in $\Delta \Omega$. Finally, the root-mean-square of the gravitational wave strain is

$$
h_{\mathrm{rms}} \approx 8 \times 10^{-28}\left(\frac{\Delta \Omega}{10 \mathrm{rad} \mathrm{s}^{-1}}\right)^{3}\left(\frac{d}{1 \mathrm{kpc}}\right)^{-1} .
$$

Figure 5 shows the peak gravitational wave predictions for the superfluid turbulence model for observed galactic pulsars and millisecond pulsars with Ro $=10^{-1}$ (black points) and $10^{-2}$ (red points). It is worth stressing that these values of the crust-core shear, $\Delta \Omega$, are most likely exaggerated; in the same paper Melatos \& Peralta (2010) showed that Ro $\lesssim 10^{-2}$ for typical millisecond pulsars. Calculations of vortex (Seveso, Pizzochero, \& Haskell 2012) and flux-tube (Link 2003) pinning suggest $\Delta \Omega$ should vary, but will generally be $\lesssim 10^{-2}$. One therefore concludes that turbulence excited in newly born protoneutron stars, or extremely close rapid rotators, are the only possible source for Advanced Era gravitational wave interferometers. The blue square and star in Figure 5 show the peak gravitational wave emission for hypothetical neutron stars situated at $d=10$ pc with spin periods $p=3 \mathrm{~ms}$ and $10 \mathrm{~ms}$, and $\mathrm{Ro}=10^{-2}$ and $10^{-1}$, respectively, the former of which would be detectable by aLIGO.

\subsection{Stochastic background}

A cosmological population of neutron stars with turbulent cores produces a stochastic gravitational wave background that peaks in aLIGOs most sensitive frequency band (Lasky, Bennett, \& Melatos 2013). Although there are large uncertainties in the expected amplitude of the background due to a lack of understanding of $\Delta \Omega$, the shape of the spectrum is relatively unique, with it being well-approximated by a piecewise power-law $\Omega_{\mathrm{gw}}\left(f_{\mathrm{gw}}\right)=\Omega_{\alpha} f_{\mathrm{gw}}^{\alpha}$, with $\alpha=7$ and $\alpha=-1$ for $f_{\mathrm{gw}}<f_{c}$ and $f_{\mathrm{gw}}>f_{c}$, respectively. Here, $f_{c}$ is the population-weighted average of $1 / \tau_{\mathrm{c}}$, and $\Omega_{\mathrm{gw}}\left(f_{\mathrm{gw}}\right)$ is the energy density in the gravitational wave background as a fraction of the closure energy density of the Universe.

It is worth noting that turbulent convection in mainsequence stars also produces a gravitational wave signal (Bennett \& Melatos 2014). Interestingly, the loudest gravitational wave signal detectable on Earth may come from the Sun as, for frequencies $f_{\mathrm{gw}} \lesssim 3 \times 10^{-4} \mathrm{~Hz}$, the Earth lies in the Sun's near zone, in which the wave strain scales significantly more steeply with distance, $h_{\mathrm{rms}} \propto d^{-5}$ (cf. $\propto d^{-1}$ in the far zone; Cutler \& Lindblom 1996; Polnarev, Roxburgh, \& Baskaran 2009). This gravitational wave signal is most relevant in the micro to $\mathrm{nHz}$ regime, and therefore most applicable to pulsar timing experiments. However, the gravitational-wave scaling at low-frequencies is uncertain as Kolmogorov scaling for turbulence breaks down below $f_{\mathrm{gw}} \lesssim 10^{-8} \mathrm{~Hz}$

\section{CONCLUSIONS}

Ground-based gravitational wave interferometers are already contributing to our understanding of interesting astrophysical phenomena. But non-detections will only progress the field so far; the first direct detection of gravitational radiation will herald a new scientific field of study, and will allow new insight into the most exotic regions of our Universe.

While the first detection with aLIGO is expected to be from the inspiral and merger of a compact binary system, there are many unknown, and ill-understood, mechanisms that can generate gravitational waves with significant amplitudes from isolated neutron stars. This review highlights many of those mechanisms where we have some understanding of the key, physical processes. It also highlights the vast uncertainty of many of these predictions, showing that they rely on knowledge of neutron star physics beyond current capabilities. But this is what makes the field so fascinating; a positive detection of gravitational waves from any of the mechanisms discussed in this paper will allow an unprecedented view into the heart of neutron stars, where some of the most exotic physics in the Universe takes place.

\section{ACKNOWLEDGEMENTS}

PDL is supported by the Australian Research Council Discovery Project DP140102578. I am extremely grateful to 
Bryn Haskell, Daniel Price, Kostas Glampedakis, and Karl Wette for useful comments on the manuscript.

\section{REFERENCES}

Aasi, J., et al. 2013, arXiv: 1304.0670

Aasi, J., et al. 2014a, ApJ, 785, 119

Aasi, J., et al. 2014b, arXiv: 1412.5942

Aasi, J., et al. 2015a, PhRvD, 91, 062008

Aasi, J., et al. 2015b, CQGra, 32, 074001

Abadie, J., et al. 2010a, ApJ, 722, 1504

Abadie, J., et al. 2010b, CQGra, 27, 173001

Abadie, J., et al. 2011a, ApJ, 734, L35

Abadie, J., et al. 2011b, PhRvD, 83, 042001

Abadie, J., et al. 2012, ApJ, 755, 2

Abbott, B., et al. 2008a, ApJ, 681, 1419

Abbott, B., et al. 2008b, CQGra, 25, 114051

Abbott, B. P., et al. 2009, ApJL., 701, L68

Abney, M., \& Epstein, R. I. 1996, JFM, 312, 327

Acernese, F., et al. 2015, CQGra, 32, 024001

Akgün, T., Reisenegger, A., Mastrano, A., \& Marchant, P. 2013, MNRAS, 433, 2445

Anderson, P. W., \& Itoh, N. 1975, Nature, 256, 25

Andersson, N. 1998, ApJ, 502, 708

Andersson, N., Comer, G. L., \& Prix, R. 2003, PhRvL, 90, 091101

Andersson, N., Jones, D. I., \& Ho, W. C. G. 2014, MNRAS, 442, 1786

Andersson, N., \& Kokkotas, K. D. 1998, MNRAS, 299, 1059

Andersson, N., \& Kokkotas, K. D. 2001, IJMPD, 10, 381

Andersson, N., Sidery, T., \& Comer, G. L. 2007, MNRAS, 381, 747

Baggio, L., et al. 2005, PhRvL, 95, 081103

Baym, G., Pethick, C., \& Pines, D. 1969, Nature, 224, 673

Bennett, M. F., \& Melatos, A. 2014, ApJ, 792, 55

Bennett, M. F., van Eysden, C. A., \& Melatos, A. 2010, MNRAS, 409, 1705

Bildsten, L. 1998, ApJL, 501, L89

Bonazzola, S., \& Gourgoulhon, E. 1996, A\&A, 312, 675

Bondarescu, R., Teukolsky, S. A., \& Wasserman, I. 2009, PhRvD, 79,104003

Braithwaite, J. 2007, A\&A, 469, 275

Braithwaite, J. 2008, MNRAS, 386, 1947

Braithwaite, J. 2009, MNRAS, 397, 763

Braithwaite, J., \& Nordlund, A. 2006, A\&A, 450, 1077

Braithwaite, J., \& Spruit, H. C. 2006, A\&A, 450, 1097

Chakrabarty, D., Morgan, E. H., Muno, M. P., Galloway, D. K., Wijnands, R., van der Klis, M., \& Markwardt, C. B. 2003, Nature, 424,42

Chandrasekhar, S. 1970, PhRvL, 24, 611

Ciolfi, R., Ferrari, V., Gualtieri, L., \& Pons, J. A. 2009, MNRAS, 397,913

Ciolfi, R., Lander, S. K., Manca, G. M., \& Rezzolla, L. 2011, ApJ, 736, L6, arXiv:1105.3971

Ciolfi, R., \& Rezzolla, L. 2012, ApJ, 760, 1

Ciolfi, R., \& Rezzolla, L. 2013, MNRAS.

Colaiuda, A., Beyer, H., \& Kokkotas, K. D. 2009, MNRAS, 396, 1441

Colaiuda, A., Ferrari, V., Gualtieri, L., \& Pons, J. A. 2008, MNRAS, 385,2080

Cook, G. B., Shapiro, S. L., \& Teukolsky, S. A. 1994, ApJ, 424, 823
Corsi, A., \& Owen, B. J. 2011, PhRvD, 83, 104014

Cutler, C. 2002, PhRvD, 66, 084025

Cutler, C., \& Lindblom, L. 1996, PhRvD, 54, 1287

Dall'Osso, S., Shore, S. N., \& Stella, L. 2009, MNRAS, 398, 1869

Detweiler, S. L. 1975, ApJ, 197, 203

Duncan, R. C., \& Thompson, C. 1992, ApJ, 392, L9

Dupuis, R. J., \& Woan, G. 2005, PhRvD, 72, 102002

Espinoza, C. M., Antonopoulou, D., Stappers, B. W., Watts, A., \& Lyne, A. G. 2014, MNRAS, 440, 2755

Espinoza, C. M., Lyne, A. G., Stappers, B. W., \& Kramer, M. 2011, MNRAS, 414, 1679

Friedman, J. L., \& Morsink, S. M. 1998, ApJ, 502, 714

Friedman, J. L., \& Schutz, B. F. 1978, ApJ, 222, 281

Gabler, M., Cerdá-Durán, P., Font, J. A., Müller, E., \& Stergioulas, N. 2011, MNRAS, 410, L37

Gabler, M., Cerdá-Durán, P., Stergioulas, N., Font, J. A., \& Müller, E. 2013, PhRvL, 111, 211102

Gabler, M., Cerdá-Durán, P., Stergioulas, N., Font, J. A., \& Müller, E. 2014, MNRAS, 443, 1416

Glampedakis, K., \& Andersson, N. 2009, PhRvL, 102, 141101

Glampedakis, K., Andersson, N., \& Lander, S. K. 2012a, MNRAS, 420, 1263

Glampedakis, K., \& Jones, D. I. 2014, MNRAS, 439, 1522

Glampedakis, K., Jones, D. I., \& Samuelsson, L. 2012b, PhRvL, 109, 081103

Glampedakis, K., \& Lasky, P. D. 2015, MNRAS, 450, 1638

Glampedakis, K., Samuelsson, L., \& Andersson, N. 2006, MNRAS, 371, L74

Greenstein, G. 1970, Nature, 227, 791

Haensel, P., \& Zdunik, J. L. 1990, A\&A, 227, 431

Hameury, J. M., Bonazzola, S., Heyvaerts, J., \& Lasota, J. P. 1983, A\&A, 128, 369

Haskell, B., Andersson, N., Jones, D. I., \& Samuelsson, L. 2007, PhRvL, 99, 231101

Haskell, B., Degenaar, N., \& Ho, W. C. G. 2012, MNRAS, 424, 93

Haskell, B., Jones, D. I., \& Andersson, N. 2006, MNRAS, 373, 1423

Haskell, B., \& Melatos, A. 2015, IJMPD, 24, 30008

Haskell, B., Priymak, M., Patruno, A., Oppenoorth, M., Melatos, A., \& Lasky, P. D. 2015, MNRAS, 450, 2393

Haskell, B., Samuelsson, L., Glampedakis, K., \& Andersson, N. 2008, MNRAS, 385, 531

Ho, W. C. G., Andersson, N., \& Haskell, B. 2011, PhRvL, 107, 101101

Huppenkothen, D., et al. 2014a, ApJ, 787, 128

Huppenkothen, D., Heil, L. M., Watts, A. L., \& Göğüş, E. 2014b, ApJ, 795, 114

Huppenkothen, D., Watts, A. L., \& Levin, Y. 2014c, ApJ, 793, 129

Ioka, K. 2001, MNRAS, 327, 639

Israel, G. L., et al. 2005, ApJ, 628, L53

Johnson-McDaniel, N. K., \& Owen, B. J. 2013, PhRvD, 88, 044004

Jones, D. I. 2010, MNRAS, 402, 2503

Jones, D. I., \& Andersson, N. 2001, MNRAS, 324, 811

Jones, D. I., \& Andersson, N. 2002, MNRAS, 331, 203

Kalmus, P., Cannon, K. C., Márka, S., \& Owen, B. J. 2009, PhRvD, 80,042001

Kashiyama, K., \& Ioka, K. 2011, PhRvD, 83, 081302

Kiuchi, K., Yoshida, S., \& Shibata, M. 2011, A\&A, 532, 17

Lander, S. K. 2013, PhRvL, 110, 071101

Lander, S. K., \& Jones, D. I. 2012, MNRAS, 424, 482 
Lasky, P. D., Bennett, M. F., \& Melatos, A. 2013, PhRvD, 87, 063004

Lasky, P. D., \& Melatos, A. 2013, PhRvD, 88, 103005

Lasky, P. D., Zink, B., \& Kokkotas, K. D. 2012, arXiv:1203.3590

Lasky, P. D., Zink, B., Kokkotas, K. D., \& Glampedakis, K. 2011, ApJ, 735, L20

Levin, Y. 2006, MNRAS, 368, L35

Levin, Y. 2007, MNRAS, 377, 159

Levin, Y., \& Lyutikov, M. 2012, MNRAS, 427, 1574

Levin, Y., \& van Hoven, M. 2011, MNRAS, 418, 659

Link, B. 2003, PhRvL, 91, 101101

Link, B. 2012a, MNRAS, 421, 2682

Link, B. 2012b, MNRAS, 422, 1640

Manchester, R. N., Hobbs, G. B., Teoh, A., \& Hobbs, M. 2005, AJ, 129, 1993

Mastrano, A., \& Melatos, A. 2005, MNRAS, 361, 927

Mastrano, A., Melatos, A., Reissenegger, A., \& Akgün, T. 2011, MNRAS, 417, 2288

McDermott, P. N., van Horn, H. M., \& Hansen, C. J. 1988, ApJ, 325,725

Melatos, A. 2012, ApJ, 761, 32

Melatos, A., Douglass, J. A., \& Simula, T. P. 2015, ApJ, 807, 132

Melatos, A., \& Payne, D. J. B. 2005, ApJ, 623, 1044

Melatos, A., \& Peralta, C. 2010, ApJ, 709, 77

Melatos, A., Peralta, C., \& Wyithe, J. S. B. 2008, ApJ, 672, 1103

Melatos, A., \& Phinney, E. S. 2001, PASA, 18, 421

Messenger, C., et al. 2015, PhRvD, 92, 023006

Mitchell, J. P., Braithwaite, J., Reisenegger, A., Spruit, H., Valdivia, J. A., \& Langer, N. 2015, MNRAS, 447, 1213

Monaghan, J. J. 1965, MNRAS, 131, 105

Monaghan, J. J. 1966, MNRAS, 134, 275

Olausen, S. A., \& Kaspi, V. M. 2014, Astrophys. J. S., 212, 6

Owen, B. J. 2005, PhRvL, 95, 211101

Owen, B. J. 2010, PhRvD, 82, 104002

Palmer, D. M., et al. 2005, Nature, 434, 1107

Papaloizou, J., \& Pringle, J. E. 1978, MNRAS, 184, 501

Patruno, A. 2010, ApJ, 722, 909

Patruno, A., Haskell, B., \& D’ Angelo, C. 2012, ApJ, 746, 9

Payne, D. J. B., \& Melatos, A. 2004, MNRAS, 351, 569

Peralta, C., Melatos, A., Giacobello, M., \& Ooi, A. 2005, ApJ, 635, 1224

Peralta, C., Melatos, A., Giacobello, M., \& Ooi, A. 2006a, ApJ, 644, L53

Peralta, C., Melatos, A., Giacobello, M., \& Ooi, A. 2006b, ApJ, 651,1079
Peralta, C., Melatos, A., Giacobello, M., \& Ooi, A. 2008, JFM, 609, 221

Polnarev, A. G., Roxburgh, I. W., \& Baskaran, D. 2009, PhRvD, 79, 082001

Priymak, M., Melatos, A., \& Lasky, P. D. 2014, MNRAS, 445, 2710

Priymak, M., Melatos, A., \& Payne, D. J. B. 2011, MNRAS, 417, 2696

Riles, K. 2013, PrPNP, 68, 1

Sammut, L., Messenger, C., Melatos, A., \& Owen, B. J. 2014, $\mathrm{PhRvD}, 89,043001$

Seveso, S., Pizzochero, P. M., \& Haskell, B. 2012, MNRAS, 427, 1089

Sidery, T., Passamonti, A., \& Andersson, N. 2010, MNRAS, 405, 1061

Sotani, H., Colaiuda, A., \& Kokkotas, K. D. 2008, MNRAS, 385, 2161

Sotani, H., Kokkotas, K. D., \& Stergioulas, N. 2007, MNRAS, 375, 261

Stella, L., Dall'Osso, S., Israel, G. L., \& Vecchio, A. 2005, ApJ, 634, L165

Strohmayer, T., \& Mahmoodifar, S. 2014, ApJ, 784, 72

Strohmayer, T. E., \& Watts, A. L. 2005, ApJ, 632, L111

Thompson, C., \& Duncan, R. C. 1995, MNRAS, 275, 255

Tsubota, M. 2009, JPCM, 21, 164207

Ushomirsky, G., Cutler, C., \& Bildsten, L. 2000, MNRAS, 319, 902

van den Broeck, C. 2005, CQGra, 22, 1825

van Eysden, C. A., \& Melatos, A. 2008, CQGra, 25, 225020

van Hoven, M., \& Levin, Y. 2011, MNRAS, 410, 1036

van Hoven, M., \& Levin, Y. 2012, MNRAS, 420, 3035

Vigelius, M., \& Melatos, A. 2009, MNRAS, 395, 1972

Wagoner, R. V. 1984, ApJ, 278, 345

Warszawski, L., \& Melatos, A. 2012, MNRAS, 423, 2058

Watts, A. L., Krishnan, B., Bildsten, L., \& Schutz, B. F. 2008, MNRAS, 389, 839

Wette, K. 2008, CQGra, 25, 235011

Whelan, J. T., Sundaresan, S., Zhang, Y., \& Peiris, P. 2015, PhRvD, 91, 102005

White, N. E., \& Zhang, W. 1997, ApJL, 490, L87

Wright, G. A. E. 1973, MNRAS, 162, 339

Zimmerman, M. 1980, PhRvD, 21, 891

Zimmerman, M., \& Szedenits, E. 1979, PhRvD, 20, 351

Zink, B., Lasky, P. D., \& Kokkotas, K. D. 2012, PhRvD, 85, 024030 